\title{
Management for Residual Ground-Glass Opan
Lesions After Resection of Main Tumor in Multifocal Lung Cancer: A Case Report and Literature Review
}

This article was published in the following Dove Press journal:

Cancer Management and Research

\author{
Bo Cheng (iD* \\ Hongsheng Deng* \\ Yi Zhao* \\ Feng Zhu (D) \\ Hengrui Liang \\ Caichen Li (DD \\ Ran Zhong \\ Jianfu Li \\ Shan Xiong \\ Zhuxing Chen \\ Wenhua Liang \\ Jianxing $\mathrm{He}$
}

Department of Thoracic Surgery and Oncology, The First Affiliated Hospital of Guangzhou Medical University, State Key Laboratory of Respiratory Disease \& National Clinical Research Center for Respiratory Disease, Guangzhou,

510120, People's Republic of China

*These authors contributed equally to this work

Correspondence: Wenhua Liang; Jianxing $\mathrm{He}$

Department of Thoracic Surgery and Oncology, The First Affiliated Hospital of Guangzhou Medical University, State Key Laboratory of Respiratory Disease \& National Clinical Research Center for Respiratory Disease, 15I, Yanjiang Road, Guangzhou, 510120, People's Republic of China

Tel +86-20-83337792

Fax +86-20-83350363

Email liangwh1987@I63.com; drjianxing. he@gmail.com
Abstract: There are increasing numbers of synchronous multiple primary lung cancer (SMPLC) patients in clinical practice, with most lesions presenting as ground-glass opacity (GGO). For SMPLC patients, surgical resection should be a prior option for all lesions suspected of being malignant, if medically and technically feasible. However, it is frequently a dilemma for the management of residual GGO lesions that were unresected simultaneously with the main tumor in SMPLC patients. We report a case of SMPLC, in which the patient underwent surgical resection of the major lesion with EGFR mutation and then received compelling EGFR-TKI treatment for one enlarging residual GGO lesion after 12 months since operation. Furthermore, a comprehensive literature review about the risk for the progress of GGOs unresected simultaneously with the main lesion and the management of these residual GGOs was also summarized. With the treatment of EGFR-TKI gefitinib for 3 months, the biggest residual GGO lesion (more than $10 \mathrm{~mm}$ ) achieved a complete response (CR), three lesions reduced in size, and the other three lesions remained stable in this case. Surgical resection for major lesion and EGFR-TKI treatment on unresected GGOs might bring favorable outcome for patients with EGFR-mutated multifocal lung cancer. This strategy is safe and effective, which could be a promising therapeutic approach for unresectable GGO lesions in EGFR-mutated SMPLC patients after primary surgery. Notably, folate receptor-positive circulating tumor cell $\left(\mathrm{FR}^{+}-\mathrm{CTC}\right)$ for therapeutic monitoring was more sensitive for GGO-featured lung adenocarcinoma than serum markers.

Keywords: multiple primary lung cancer, ground-glass opacity, epidermal growth factor receptor-tyrosine kinases inhibitor, folate receptor-positive circulating tumor cell, literature review

\section{Plain Language Summary}

Previous studies had revealed that chemotherapy and anti-PD-1/PD-L1-based treatment were not sensitive to the lung cancer presenting as ground-glass opacity (GGO) in computed tomography (CT). Notably, in our reported case, EGFR-TKI showed activity on unresected GGO lesions in an EGFR-mutated multiple primary lung cancer (MPLC) patient.

In this study, we presented a novel strategy for the treatment of a case with EGFR-mutant multifocal lung cancer and had multiple GGO lesions remained after the initial operation. We used surgical resection for the major lesion which was the most invasive, and continued gefitinib treatment for unresectable GGO lesions, avoiding the invasive examination like biopsy. Surprisingly, with the treatment of EGFR-TKI gefitinib for 3 months, the enlarging unresected 
GGO lesion (more than $10 \mathrm{~mm}$ ) achieved a complete response. So far, this patient is still alive and has achieved remission for 8 months. We believe this strategy is safe and effective, which could be a promising approach for the treatment of the residual GGO lesions in MPLC patients after resection of main tumor, especially for those who were unsuitable or unwilling to receive one more operation.

\section{Introduction}

Ground-glass opacity (GGO), defined as a hazy area of increased attenuation in the chest $\mathrm{CT}$, can be caused by various diseases such as interstitial diseases, inflammation, fibrosis, or neoplastic proliferation of lung. ${ }^{1}$ Synchronous multiple GGOs are defined as $\geq 2$ GGO lesions found at the same time in a single patient, ${ }^{2}$ which is increasingly detected with use of high-resolution computed tomography (CT) scan. ${ }^{3}$ As reported in previous clinical series, around $20-30 \%$ of GGO lesions resected were found to be accompanied by multiple other less invasive GGO lesions. ${ }^{4}$ For patients with synchronous multiple primary lung cancer (SMPLC) presenting as multifocal GGOs, it is always a dilemma to simultaneously remove all lesions ${ }^{5}$ and the management of the unresected GGOs after resection of the major tumor remains controversial. One routine option is to resect the major lesion(s), followed by selective resection or surveillance of the remaining lesions. ${ }^{5,6}$ However, in some conditions, surgical therapy on residual GGO lesions was unfeasible for those patients with poor pulmonary function and tolerance after resection of the major tumor. Therefore, an effective intervention was essential for SMPLC patients with high risk of progression of unresected lesions, especially for those who were unsuitable or unwilling to receive one more operation.

In the present study, we reported a case of SMPLC, in which the patient underwent surgical resection of the major lesion harboring EGFR mutation and then received EGFR-TKI treatment for one enlarging residual GGO lesion after 12 months since the initial operation, with a favorable outcome observed. In addition, a comprehensive literature review about the risk for the progress of GGOs which were unresected simultaneously with the main tumor, and the management of these residual GGOs in SMPLC patients was summarized.

\section{Case Presentation}

In September 2018, a 66-year-old asymptomatic neversmoker male was admitted to our hospital because of bilateral lung GGO lesions, which were initially detected on a routine health checkup 4 years ago. This patient received the treatment of traditional Chinese medicine for nearly 2 years, while his bilateral lung lesions had no changes in previous CT checkups. The chest CT (1mm thickness) revealed two irregularly shaped GGOpredominant lesions in the left lower lobe (LLL) and left upper lobe (LUL) of the lung which measured $18 \times 10 \mathrm{~mm}$ and $11 \times 8 \mathrm{~mm}$, respectively, and six GGO lesions of variable sizes $(3-9 \mathrm{~mm})$ in all lobes of both lungs except the right middle lobe (Figure 1A and B). Mediastinal and hilar lymph nodes did not enlarge.

The medical history of this patient included hypertension and coronary heart disease. Additionally, his sister was diagnosed definitely with lung adenocarcinoma 5 years ago, harboring EGFR L858R mutation at exon 21. Considering his bilateral multiple lung nodules, it was difficult to remove all lesions simultaneously in this patient. Systemic general examination including abdomenthorax CT and brain MRI, etc, was done for exclusion of distant metastasis, evaluating surgical possibility and there was nothing abnormal found. Afterwards, this patient underwent LLL lobectomy and mediastinal lymph node dissections through a biportal video-assisted thoracic operation. Histopathologic examination showed a $12 \times 6 \times 4 \mathrm{~mm}$ minimally invasive adenocarcinoma (MIA) of the LLL and the invasive range of tumor was about $4 \mathrm{~mm}$. All resected lymph nodes were negative. A 20mutations-panel genetic test included EGFR et al by means of next-generation sequencing indicated the EGFR L858R mutation at exon 21, with an abundance of $9.97 \%$. Combining his histopathologic examination results of stage I lung cancer (T1bN0M0, MIA) and clinical character that these GGO lesions in his bilateral lung were located in different lobes, he was diagnosed as synchronous multiple primary lung cancer according to the $\mathrm{ACCP}^{7}$ and IASLC ${ }^{2}$ criteria. After discharge, close postoperative surveillance and regular CT checkup were conducted for the remained GGO lesions.

In March 2019, 6 months after surgery, this patient underwent his first postoperative CT reexamination, showing that the LUL GGO lesion enlarged to $13 \times 9 \mathrm{~mm}$ and other lesions had no changes in size. Meanwhile, he received the detection of folate receptor-positive circulating tumor cell $\left(\mathrm{FR}^{+}-\mathrm{CTC}\right)$ on his own, and the $\mathrm{FR}+-\mathrm{CTC}$ count was $6.2 \mathrm{FU} / 3 \mathrm{~mL}$ (the reference value was $<8.7 \mathrm{FU} /$ $3 \mathrm{~mL})$. Afterwards, he did not receive any treatment and continued to follow up for 6 months until his FR+-CTC count was much higher than the reference value in 


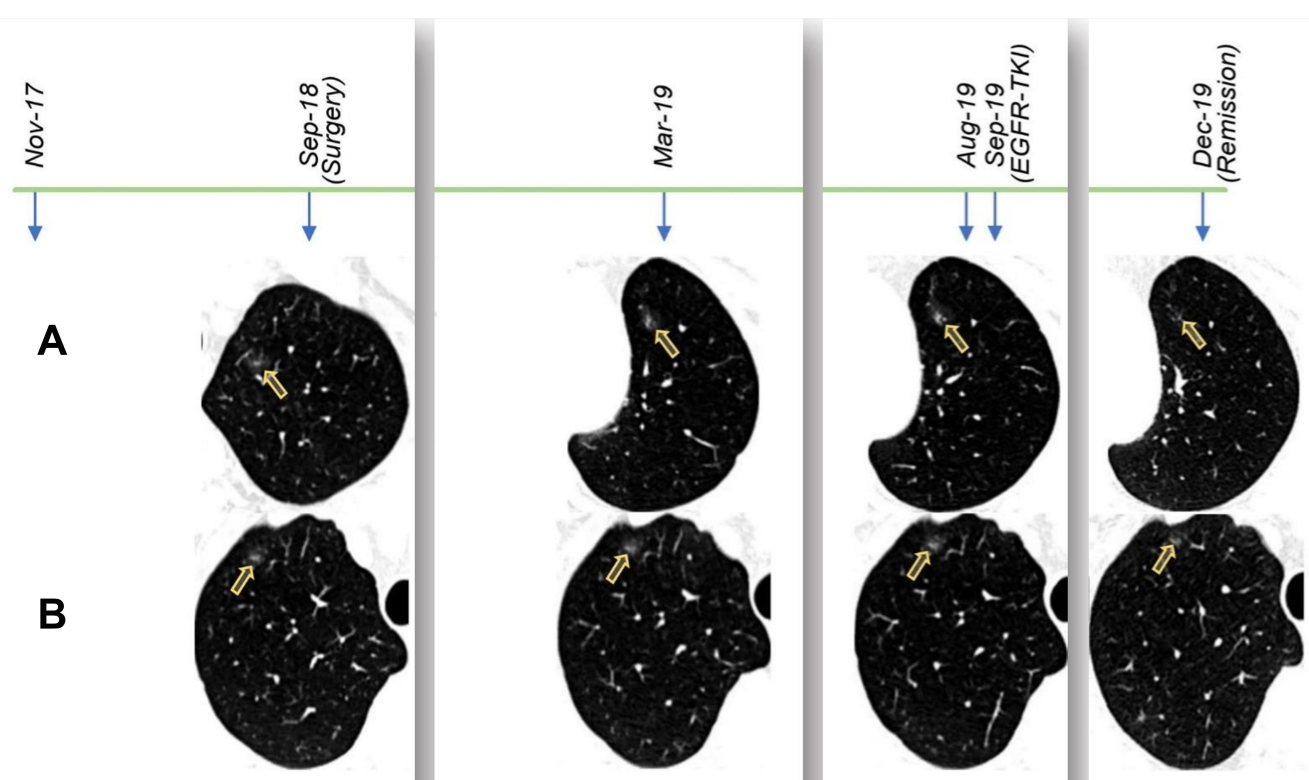

C

25

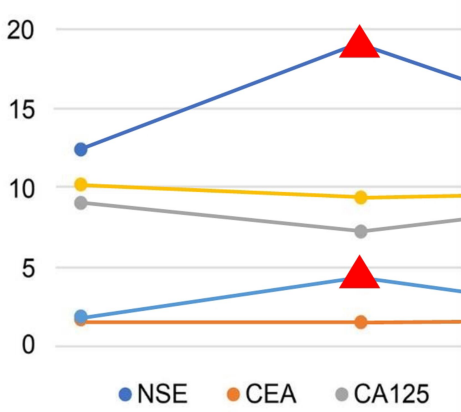

D

35

30

25

20

15

10

5

0

Reference value

Higher than reference value

\section{Serum Tumor Marker}
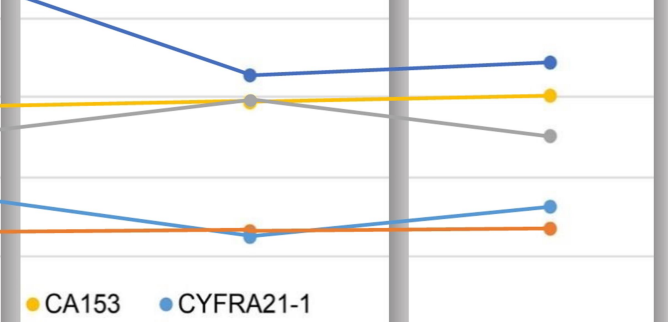

FR+-CTC Count

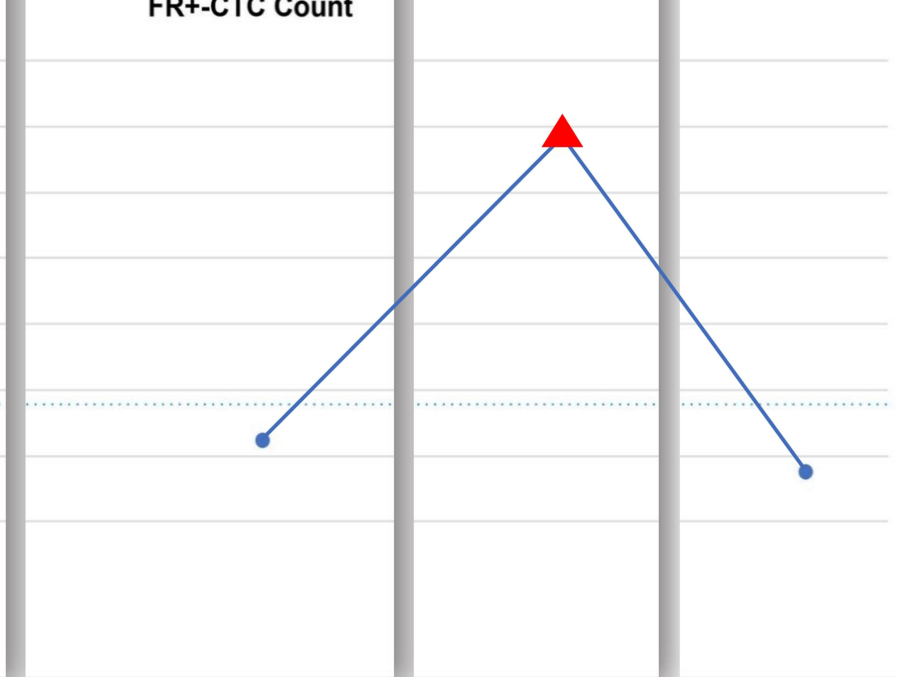

Figure I The examination results of CT, serum tumor marker and FR+-CTC were described in chronological order, included the time before operation, follow-up without medication and after EGFR-TKI treatment. (A and B). CT image of unresected GGO lesions in left upper lobe (LUL) and right upper lobe (RUL) in patients, showing the enlargement and remission of lesions. The yellow arrows in $\mathbf{A}$ and $\mathbf{B}$ pointed to the location of lesions in the CT images. (C and $\mathbf{D})$. Line chart showed the changes in value of serum tumor marker and FR+-CTC in different periods of time. 
examination $(29.2 \mathrm{FU} / 3 \mathrm{~mL})$ in August 2019 and without any changes in CT image during this period. Notably, except the result of preoperational detection was higher than the reference value for once, all serum tumor markers value of this patient included NSE and CEA et al were persistently within normal value during the whole followup (Figure $1 \mathrm{C}$ and D).

Thus, in September 2019, this patient came to our department for treatment again. In comprehensive consideration of his medical history and all check-up results included $\mathrm{CT}$ and $\mathrm{FR}^{+}$-CTC, we suggested him to undergo the treatment of first-generation EGFR-TKI gefitinib (250mg, once daily). In December 2019, the patient underwent the examination of $\mathrm{CT}$ and $\mathrm{FR}+-\mathrm{CTC}$ again after the treatment of gefitinib for 3 months. Surprisingly, we found that the LUL lesion achieved complete response (CR) in terms of the RECIST criteria, ${ }^{8}$ three lesions had a reduction in size and the other three lesions remained with no changes in size. Furthermore, his FR+-CTC count was $3.8 \mathrm{FU} / 3 \mathrm{~mL}$, which reached the lowest value in three times of examination and was significantly lower than the reference value. This patient is currently receiving maintenance gefitinib treatment and has been in remission for 8 months, without any adverse events observed.

\section{Discussion and Literature Review}

In terms of the natural history of GGOs, larger tumor size (diameter larger than $10 \mathrm{~mm}$ ), ${ }^{9}$ greater CT attenuation, ${ }^{10}$ or various specific structural features (bubble lucency ${ }^{11}$ and solid proportion ${ }^{12}$ ) are associated with risk of progression. If medically and technically feasible, surgical resection should be performed for all lesions suspected of being malignant, as an effective treatment approach for SMPLC patients. ${ }^{10}$ However, it is unfeasible to remove all lesions in this case and the biggest enlarged GGO lesion was located in left lung that has undergone lobectomy for once. Therefore, EGFR-TKI therapy as a novel treatment strategy was adopted on unresected GGO lesions of this patient, which was mainly based on the three following points: 1) The resected lesion in LLL indicated sensitive EGFR mutation with gene test; 2) The biggest residual GGO lesion in LUL showed enlargement in size at the first-time CT check-up after surgery; 3) $\mathrm{FR}^{+}$-CTC count of this patient performed much higher than the reference value, which revealed that there were active malignant lesions in the patient. Notably, this patient achieved a CR of the LUL lesion and significant remission of other unresected lesions.

For SMPLC patients, it is still a dilemma for the management of residual GGO lesion(s), which were not resected simultaneously with the main tumor in the initial surgery. When there is uncertainty, personal opinion and experience should not influence decision-making, on the contrary decision should be taken by a multidisciplinary team containing the thoracic surgeon, oncologist, radiologist and so on. ${ }^{13}$ According to Kobayashi et al, ${ }^{14}$ approximately $20 \%$ of pure GGOs and $40 \%$ of part-solid GGOs gradually increased with the size or solid component. In a series of studies reporting the fate unresected GGO lesions with the duration of follow-up $\geq 2$ years, the progressed rate ranged from $0 \%$ to $32.8 \% .^{3,5,15-18}$ Therefore, close postoperative surveillance was essential for these patients harboring unresected GGO lesions. Publications reporting the management and fate of unresected GGO lesions in SMPLC patients are summarized in Table 1.

Various studies have detailed the treatment strategy of SMPLC. Shimada et $\mathrm{al}^{3}$ noted that for multiple GGOdominant lesions scattered throughout multiple lobes, surgical resection for the main lesion and easily accessible solid nodules by limited resection is preferred. Stereotactic body radiation therapy (SBRT) or continuous monitoring throughout the postoperative follow-up period is adopted for lesions with a diameter not larger than $10 \mathrm{~mm}$, or without an emerging solid portion. Recently, Shimada et $\mathrm{al}^{18}$ reported that 38 out of 116 SMPLC patients harboring unresected GGOs occurred progression after the initial surgery, and ultimately 30 underwent surgery, 1 had radiotherapy and the rest 7 remained in surveillance. Gao et al ${ }^{16}$ retrospectively analyzed the treatment outcomes in 70 patients with unresected GGOs after resection of a pN0 dominant tumor, with 5 (7.1\%) underwent surgical resection and $6(8.6 \%)$ received stereotactic radiotherapy. In general, continuous monitoring plus surgery or SBRT when the unresected GGOs performed progression was currently a frequent treatment strategy for SMPLC patients. However, a considerable proportion of SMPLC patients were unfeasible to receive further surgical therapy on unresected GGO after their primary operation, due to the poor pulmonary reserve or tolerance (such as advanced age or comorbidities). Besides, numerous patients prefer conservative treatment to surgery or radiotherapy in clinical practice, considering the body-invasion and complications. ${ }^{19,20}$ Therefore, an effective and noninvasive intervention was needful for these patients with 


\begin{tabular}{|c|c|c|c|c|c|c|c|}
\hline 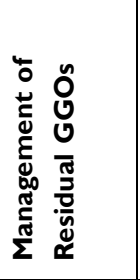 & 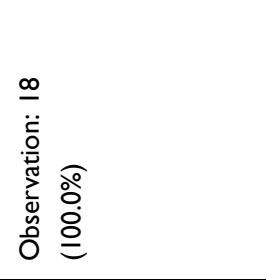 & 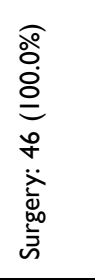 & 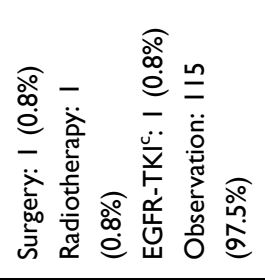 & 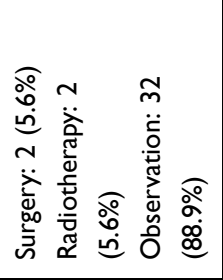 & 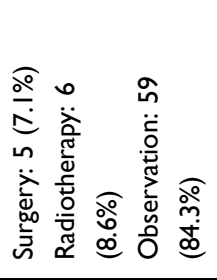 & 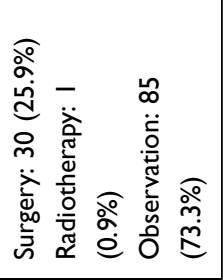 & 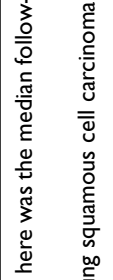 \\
\hline 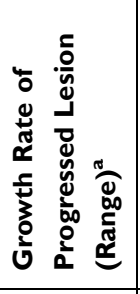 & $\mathbb{z}$ & 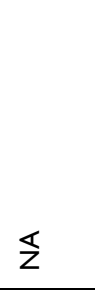 & 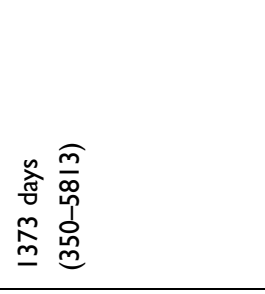 & 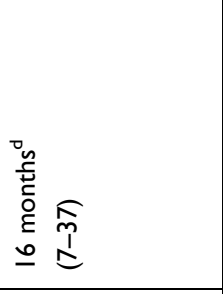 & 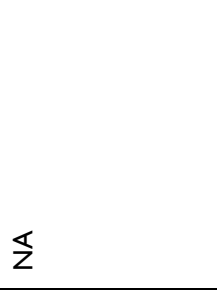 & 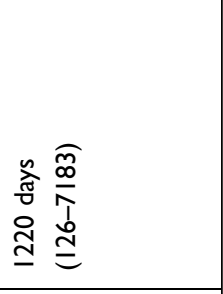 & 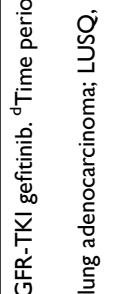 \\
\hline 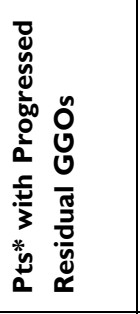 & 0 & 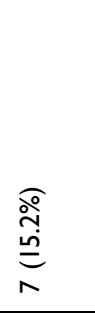 & 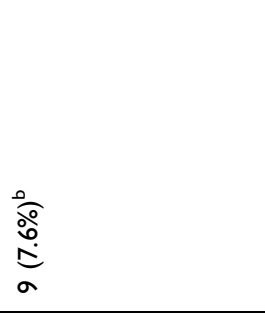 & 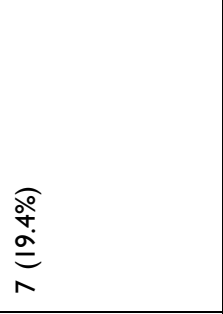 & $\begin{array}{l}\frac{0}{a} \\
\stackrel{d}{a} \\
\frac{a}{2}\end{array}$ & 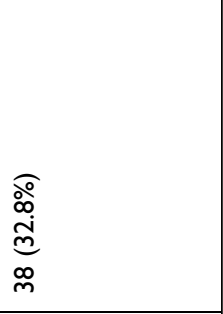 & 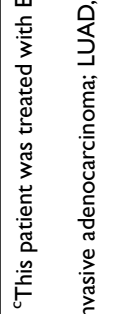 \\
\hline 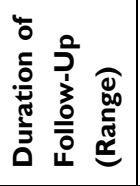 & 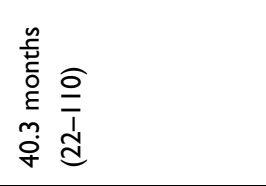 & 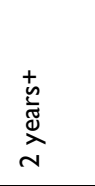 & 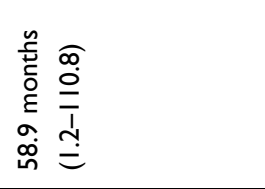 & 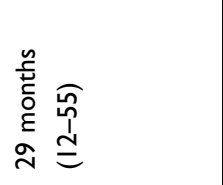 & 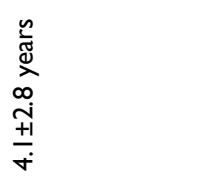 & 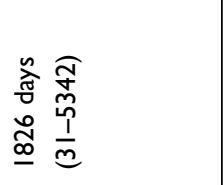 & 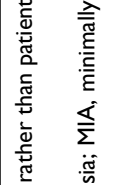 \\
\hline 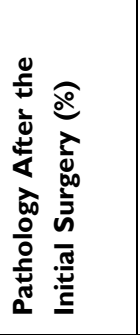 & 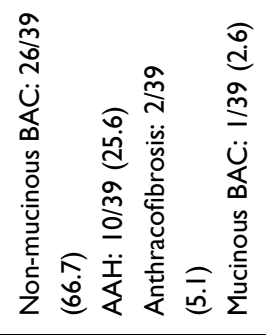 & 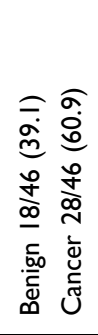 & 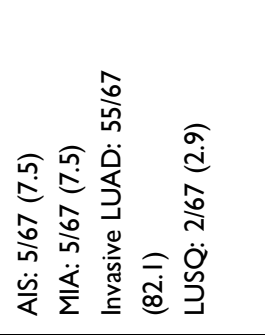 & 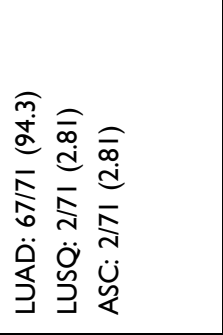 & 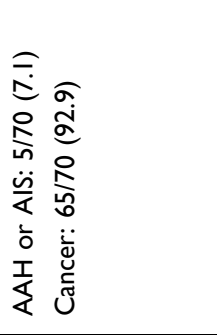 & 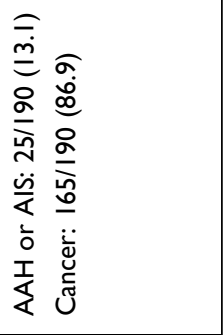 & 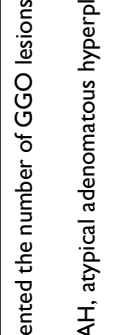 \\
\hline 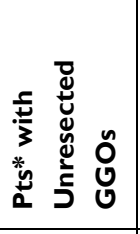 & $\underline{\infty}$ & $\stackrel{+}{+}$ & $\stackrel{\infty}{=}$ & $\stackrel{m}{m}$ & $R$ & $\stackrel{\infty}{=}$ & 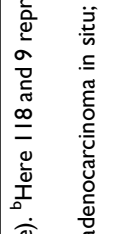 \\
\hline 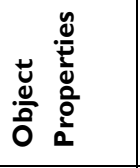 & $\begin{array}{l}\text { O } \\
\text { O } \\
\end{array}$ & 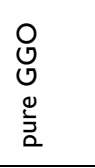 & $\begin{array}{l}0 \\
\text { O } \\
\end{array}$ & 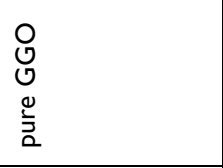 & $\begin{array}{l}\text { O } \\
\text { ○ }\end{array}$ & $\begin{array}{l}\text { O } \\
\text { O }\end{array}$ & 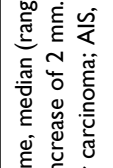 \\
\hline 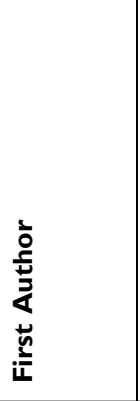 & 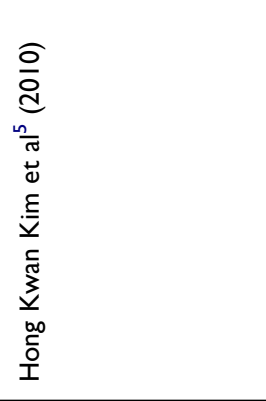 & 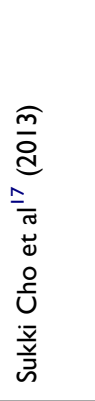 & 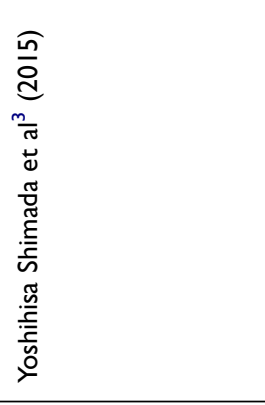 & 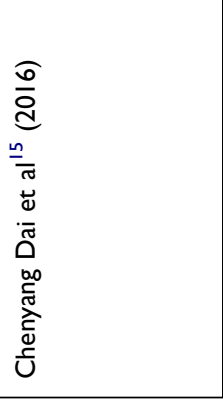 & 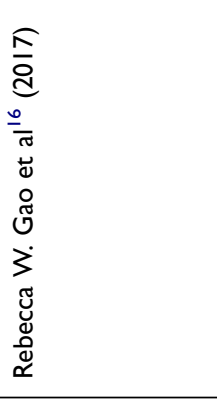 & 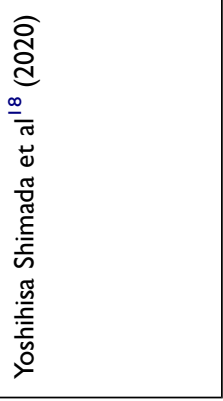 & 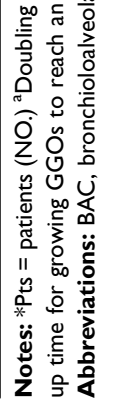 \\
\hline
\end{tabular}


a high risk of progression of unresected lesions, especially for these who were unsuitable or unwilling to receive one more operation.

So far, there are no established guidelines of the adjuvant or systemic treatment for SMPLC. Finley et $\mathrm{al}^{21}$ reported results of 175 patients that underwent surgical resection for synchronous multiple GGOs. Twenty-five patients (14.3\%) were given adjuvant therapy; 17 received chemotherapy and 8 underwent external radiotherapy. However, Zhang et $\mathrm{al}^{22}$ retrospectively included patients who had GGO-featured lung cancer before undergoing chemotherapy for other concurrent malignancies; eventually, all the GGO lesions either remained unchanged or enlarged and no chemotherapy-induced histopathologic response (necrosis, fibrosis or inflammation) was observed in any of the resected GGO lesions. Similarly, $\mathrm{Lu}$ et $\mathrm{al}^{23}$ evaluated the response of persistent GGOs in lung adenocarcinoma patients treated with platinum-based chemotherapy and concluded that GGOs were insensitive to the chemotherapy.

In recent years, immune checkpoint inhibitors (ICIs) targeting programmed cell death protein 1 (PD-1) and its ligand (PD-L1) have been proven its significant efficacy on advanced non-small cell lung cancer (NSCLC). ${ }^{24,25}$ Jiang et $\mathrm{al}^{26}$ observed the effect of anti-PD-1/PD-L1based therapy on 37 synchronous GGOs from 18 advanced lung adenocarcinoma (LUAD) patients. However, only three mixed GGOs (8.1\%) showed response to the antiPD-1/PD-L1-based treatment, indicating that GGOs may not be sensitive to anti-PD-1/PD-L1-based monotherapy or combinatorial therapy. In 2020, Liang et al reported a case in which the perioperative chemotherapy combined with anti-PD-1 immunotherapy led to a good survival benefit in a patient with stage IIIB NSCLC. ${ }^{27}$ However, whether the chemoimmunotherapy shows effect on early-stage lung cancer presenting as GGO lesions was unclear and worth exploring in the future studies.

Currently, few studies reported the potency of molecular targeted therapy on GGO lesions of lung cancer, but some cases demonstrated the favorable efficacy of EGFRTKIs on GGOs in EGFR-mutated SMPLC patients. In 2016, Ye et al ${ }^{28}$ reported a case of SMPLC who underwent surgical resection of the gefitinib-insensitive peripheral lesion and continued gefitinib treatment for the residual gefitinib-sensitive GGO lesions. This patient achieved CR and has been free of disease for 1.5 years. Similarly, in our present case, radical resection for all bilateral multiple GGO lesions was not feasible for this patient; thus, EGFR-
TKI was administrated for treatment of the enlarging malignant GGO lesion. This case helped emphasize the role of EGFR-TKI and provide a non-invasive therapeutic approach for unresected GGO lesions in EGFR-mutated SMPLC patients. Previous multicenter trials ${ }^{29-31}$ have released the latest data of adjuvant EGFR-TKIs in patients with completely resected EGFR-mutant NSCLC. Notably, ADAURA trial published in $N E J M^{29}$ confirmed that for patients with early-stage (IB-IIIA) resected EGFR-mutant NSCLC, adjuvant EGFR-TKIs led to significantly longer disease-free survival (DFS) compared with that for chemotherapy. Recently, osimertinib was recommended as an adjuvant regiment for resected stage IB-IIIA NSCLC patients by NCCN guideline. ${ }^{32}$ Therefore, for these EGFRmutated MPLC patients harboring earlier-stage unresectable tumor presenting as GGO lesions, with more prospective studies conducted and proved, the therapy of EGFRTKIs might be a potential drug covered by medical insurance in the future at worldwide.

Screening and monitoring of GGOs and small lesions is a problem in clinical practice. The maximum standardized uptake value (SUVmax) in PET/CT is closely related to cancer proliferation, progression, and metastasis, and its value reflects the invasiveness of tumors. ${ }^{33}$ Previous literatures ${ }^{34}$ have demonstrated that lesions with invasive CT morphologic features, such as lobulation sign, pleural indentation sign, and vascular convergence sign were prone to have higher SUVmax than that of GGOs without these signs. However, the sensitivity of PET/CT for differentiating the malignancy of GGOs might not be adequate. Kim et $\mathrm{al}^{35}$ reported that in a cohort with multiple GGO-predominant lesions, only 27\% (21/78) among these GGOs showed hypermetabolism. In addition, adenocarcinoma with bronchioloalveolar carcinoma (BAC) features was considered as the main cause of false-negative finding on PET/CT in Wu et al study. ${ }^{36}$ Therefore, the necessity of PET/CT in early-stage lung cancer presenting as GGOs was still under debate and several recent studies have demonstrated that PET/CT did not appear to offer a clear advantage in lung cancer manifesting as predominant GGOs because of low prevalence of lymph nodes or distant metastasis. ${ }^{37,38}$

In comparison with the PET/CT and serum tumor marker, $\mathrm{FR}^{+}$-CTC seemed a more sensitive biomarker to reveal the tumor burden in early-stage lung cancer. In brief, CTCs were negatively enriched from the $3 \mathrm{~mL}$ of blood sample by immunomagnetic depletion of leukocytes and then labeled with the proprietary probe (conjugates of 
a tumor-specific folic acid ligand and a synthesized oligonucleotide). The procedure of $\mathrm{FR}+\mathrm{CTC}$ analysis was in substantial agreement as mentioned in other publications. ${ }^{39-42}$ Previous studies ${ }^{39-44}$ demonstrated that $\mathrm{FR}^{+}$-CTC level in the peripheral blood could differentiate benign from malignant pulmonary nodules with a sensitivity of $73.2 \%-82.7 \%$ and a specificity of $68.8 \%$ $89.5 \%$, which showed a higher diagnostic efficiency compared with the serum tumor markers, indicating that the $\mathrm{FR}^{+}$-CTC could be a feasible diagnostic biomarker in patients with NSCLC. Additionally, the $\mathrm{FR}^{+}$-CTC detection was useful for the determination of the invasiveness of lung nodules preoperatively, ${ }^{42}$ the surveillance of NSCLC patients after tumor resection, ${ }^{39}$ the dynamic monitoring in patients treated with anti-tumor drugs, ${ }^{44}$ as a supplement or alternative to CT scanning. Therefore, we reasonably considered that the $\mathrm{FR}^{+}$-CTC detection is a reliable method for the diagnosis and monitoring of GGOs.

\section{Conclusion}

Previous studies had revealed that chemotherapy and antiPD-1/PD-L1-based treatment were not sensitive to the GGO lesions. Notably, in our reported case, EGFR-TKI showed activity on unresected GGO lesions in an EGFRmutated SMPLC patient.

In conclusion, we presented a novel strategy for the treatment of a case with EGFR-mutant SMPLC and had multiple GGO lesions remained after the initial operation. We used surgical resection for the major lesion which was the most invasive, and continued gefitinib treatment for unresectable GGO lesions, avoiding the invasive examination like biopsy. What is more, close CT check-up played an important role in the surveillance of tumor and the FR +-CTC seemed a promising biomarker to reveal the tumor burden in this case and needed to be further validated prospectively. So far, this patient is still alive and has achieved remission for 8 months. We believe this strategy is safe and effective, which could be a promising approach for the treatment of unresected GGO lesions in SMPLC patients after resection of the main tumor, especially for those who were unsuitable or unwilling to receive one more operation.

\section{Abbreviations}

SMPLC, synchronous multiple primary lung cancer; GGO, ground-glass opacity; CR, complete response; FR+CTC, folate receptor-positive circulating tumor cell; CT, computed tomography; LLL, left lower lobe; LUL, left upper lobe; ICIs, immune checkpoint inhibitors; PD-1, programmed cell death protein 1; NSCLC, non-small cell lung cancer; LUAD, lung adenocarcinoma; MIA, minimally invasive adenocarcinoma.

\section{Data Sharing Statement}

The data that support the findings of this study are available on request from the corresponding author Jianxing He, Email: drjianxing.he@gmail.com.

\section{Consent for Publication}

The authors have obtained informed consent from the patient for publication of this case report, and the ethics committee of The First Affiliated Hospital of Guangzhou Medical University approved this consent process and the publication of case details.

\section{Acknowledgments}

Bo Cheng, Hongsheng Deng, and Yi Zhao are co-first authors for this study.

\section{Author Contributions}

All authors made a significant contribution to the work reported, whether that is in the conception, study design, execution, acquisition of data, analysis and interpretation, or in all these areas; took part in drafting, revising or critically reviewing the article; gave final approval of the version to be published; have agreed on the journal to which the article has been submitted; and agree to be accountable for all aspects of the work.

\section{Disclosure}

The authors declared no conflicts of interest.

\section{References}

1. Park CM, Goo JM, Lee HJ, Lee CH, Chun EJ, Im J-G. Nodular ground-glass opacity at thin-section CT: histologic correlation and evaluation of change at follow-up. Radiographics. 2007;27(2): 391-408. doi:10.1148/rg.272065061

2. Detterbeck FC, Marom EM, Arenberg DA, et al. The IASLC lung cancer staging project: background data and proposals for the application of TNM staging rules to lung cancer presenting as multiple nodules with ground glass or lepidic features or a pneumonic type of involvement in the Forthcoming Eighth Edition of the TNM classification. J Thoracic Oncol. 2016;11:666-680.

3. Shimada Y, Saji H, Otani K, et al. Survival of a surgical series of lung cancer patients with synchronous multiple ground-glass opacities, and the management of their residual lesions. Lung Cancer (Amsterdam, Netherlands). 2015;88:174-180. 
4. Jiang G, Xie D. [Early-stage lung cancer manifested as ground-glass opacity]. Zhonghua Wai Ke Za Zhi [Chinese Journal of Surgery]. 2015;53:790-793. Chinese.

5. Kim HK, Choi YS, Kim J, Shim YM, Lee KS, Kim K. Management of multiple pure ground-glass opacity lesions in patients with bronchioloalveolar carcinoma. J Thoracic Oncol. 2010;5:206-210.

6. Loukeri AA, Kampolis CF, Ntokou A, Tsoukalas G, Syrigos K. Metachronous and synchronous primary lung cancers: diagnostic aspects, surgical treatment, and prognosis. Clin Lung Cancer. 2015;16:15-23.

7. Kozower BD, Larner JM, Detterbeck FC, Jones DR. Special treatment issues in non-small cell lung cancer: diagnosis and management of lung cancer, 3rd ed: American College of Chest Physicians evidence-based clinical practice guidelines. Chest. 2013;143:e369Se399S.

8. Eisenhauer EA, Therasse P, Bogaerts J, et al. New response evaluation criteria in solid tumours: revised RECIST guideline (version 1.1). Eur J Cancer. 2009;45:228-247.

9. Chang B, Hwang JH, Choi Y-H, et al. Natural history of pure ground-glass opacity lung nodules detected by low-dose CT scan. Chest. 2013;143:172-178.

10. Tamura M, Shimizu Y, Yamamoto T, Yoshikawa J, Hashizume Y. Predictive value of one-dimensional mean computed tomography value of ground-glass opacity on high-resolution images for the possibility of future change. $J$ Thoracic Oncol. 2014;9(4):469-472. doi:10.1097/JTO.0000000000000117

11. Lee SW, Leem C-S, Kim TJ, et al. The long-term course of ground-glass opacities detected on thin-section computed tomography. Respir Med. 2013;107:904-910.

12. Li M, Wu N, Zhang L, et al. Solid component proportion is an important predictor of tumor invasiveness in clinical stage TNM (cTNM) lung adenocarcinoma. Cancer Imaging. 2018;18:18.

13. Migliore M, Fornito M, Palazzolo M, et al. Ground glass opacities management in the lung cancer screening era. Ann Transl Med. 2018;6:90.

14. Kobayashi Y, Mitsudomi T. Management of ground-glass opacities: should all pulmonary lesions with ground-glass opacity be surgically resected? Transl Lung Cancer Res. 2013;2:354-363.

15. Dai C, Ren Y, Xie H, et al. Clinical and radiological features of synchronous pure ground-glass nodules observed along with operable non-small cell lung cancer. J Surg Oncol. 2016;113:738-744.

16. Gao RW, Berry MF, Kunder CA, et al. Survival and risk factors for progression after resection of the dominant tumor in multifocal, lepidic-type pulmonary adenocarcinoma. $J$ Thorac Cardiovasc Surg. 2017;154.

17. Cho S, Yang H, Kim K, Jheon S. Pathology and prognosis of persistent stable pure ground-glass opacity nodules after surgical resection. Ann Thorac Surg. 2013;96:1190-1195.

18. Shimada Y, Maehara S, Kudo Y, et al. Profiles of lung adenocarcinoma with multiple ground-glass opacities and the fate of residual lesions. Ann Thorac Surg. 2020;109:1722-1730.

19. Sun X, Li Y, Zhu Y, et al. Four-year follow-up outcomes after stereotactic body radiation therapy for central early-stage non-small cell lung cancer. Transl Lung Cancer Res. 2020;9:1472-1482.

20. Eriguchi T, Takeda A, Sanuki N, et al. Stereotactic body radiotherapy for operable early-stage non-small cell lung cancer. Lung Cancer (Amsterdam, Netherlands). 2017;109:62-67.

21. Finley DJ, Yoshizawa A, Travis W, et al. Predictors of outcomes after surgical treatment of synchronous primary lung cancers. $J$ Thoracic Oncol. 2010;5:197-205.

22. Zhang Y, Deng C, Ma X, et al. Ground-glass opacity-featured lung adenocarcinoma has no response to chemotherapy. J Cancer Res Clin Oncol. 2020;146:2411-2417.

23. $\mathrm{Lu} \mathrm{W}$, Cham QL, Wang J, et al. The impact of chemotherapy on persistent ground-glass nodules in patients with lung adenocarcinoma. J Thorac Dis. 2017;9:4743-4749.
24. Mok TSK, Wu Y-L, Kudaba I, et al. Pembrolizumab versus chemotherapy for previously untreated, PD-L1-expressing, locally advanced or metastatic non-small-cell lung cancer (KEYNOTE-042): a randomised, open-label, controlled, Phase 3 trial. Lancet (London, England). 2019;393:1819-1830.

25. Antonia SJ, Villegas A, Daniel D, et al. Overall survival with durvalumab after chemoradiotherapy in Stage III NSCLC. $N$ Engl J Med. 2018;379:2342-2350.

26. Wu F, Li W, Zhao W, et al. Synchronous ground-glass nodules showed limited response to anti-PD-1/PD-L1 therapy in patients with advanced lung adenocarcinoma. Clin Transl Med. $2020 ; 10$.

27. Liang H, Deng H, Liang W, et al. Perioperative chemoimmunotherapy in a patient with stage IIIB non-small cell lung cancer. Ann Transl Med. 2020;8:245.

28. Ye C, Wang J, Li W, Chai Y. Novel strategy for synchronous multiple primary lung cancer displaying unique molecular profiles. Ann Thorac Surg. 2016;101:e45-e47.

29. Wu Y-L, Tsuboi M, He J, et al. Osimertinib in resected EGFRmutated non-small-cell lung cancer. $N$ Engl J Med. 2020.

30. Zhong W-Z, Wang Q, Mao W-M, et al. Gefitinib versus vinorelbine plus cisplatin as adjuvant treatment for stage II-IIIA (N1-N2) EGFR-mutant NSCLC (ADJUVANT/CTONG1104): a randomised, open-label, phase 3 study. Lancet Oncol. 2018;19:139-148.

31. Yue D, Xu S, Wang Q, et al. Erlotinib versus vinorelbine plus cisplatin as adjuvant therapy in Chinese patients with stage IIIA EGFR mutation-positive non-small-cell lung cancer (EVAN): a randomised, open-label, Phase 2 trial. Lancet Respir Med. 2018;6:863-873.

32. Ettinger DSWD, Aggarwal C NCCN Clinical Practice Guidelines in Oncology: non-Small Cell Lung Cancer. Version 2.2021. 2020.

33. Takenaka $\mathrm{T}$, Yano $\mathrm{T}$, Ito $\mathrm{K}$, et al. Biological significance of the maximum standardized uptake values on positron emission tomography in non-small cell lung cancer. J Surg Oncol. 2009;10 0:688-692.

34. Shao X, Niu R, Jiang Z, Shao X, Wang Y. Role of PET/CT in Management of early lung adenocarcinoma. AJR Am J Roentgenol. 2020;214:437-445.

35. Kim TJ, Park CM, Goo JM, Lee KW. Is there a role for FDG PET in the management of lung cancer manifesting predominantly as ground-glass opacity? AJR Am J Roentgenol. 2012;198:83-88.

36. Wu HB, Wang L, Wang QS, et al. Adenocarcinoma with BAC features presented as the nonsolid nodule is prone to be false-negative on 18F-FDG PET/CT. Biomed Res Int. 2015;2015:243681.

37. Liang W, Zhao Y, Huang W, et al. Non-invasive diagnosis of early-stage lung cancer using high-throughput targeted DNA methylation sequencing of circulating tumor DNA (ctDNA). Theranostics. 2019;9:2056-2070.

38. Baisi A, Raveglia F, Cioffi U. Preoperative positron emission tomography/computed tomography in pulmonary ground glass opacities: a useful diagnostic and staging tool or not? $J$ Thorac Cardiovasc Surg. 2016;151:279-280.

39. Wang L, Wu C, Qiao L, et al. Clinical significance of folate receptor-positive circulating tumor cells detected by ligand-targeted polymerase chain reaction in lung cancer. J Cancer. 2017;8:104-110.

40. Chen X, Zhou F, Li X, et al. Folate receptor-positive circulating tumor cell detected by LT-PCR-based method as a diagnostic biomarker for non-small-cell lung cancer. $J$ Thorac Oncol. 2015;10:1163-1171.

41. Xue Y, Cong W, Xie S, Shu J, Feng G, Gao H. Folate-receptorpositive circulating tumor cells as an efficacious biomarker for the diagnosis of small pulmonary nodules. $J$ Cancer Res Ther. 2018;14:1620-1626. 
42. Zhou Q, Geng Q, Wang L, et al. Value of folate receptor-positive circulating tumour cells in the clinical management of indeterminate lung nodules: a non-invasive biomarker for predicting malignancy and tumour invasiveness. EBioMedicine. 2019;41:236-243.

43. Yu Y, Chen Z, Dong J, et al. Folate receptor-positive circulating tumor cells as a novel diagnostic biomarker in non-small cell lung cancer. Transl Oncol. 2013;6:697-702.
44. Jiang T, Zhao J, Zhao C, et al. Dynamic monitoring and predictive value of circulating tumor cells in EGFR-mutated advanced non-small-cell lung cancer patients treated with first-line EGFR tyrosine kinase inhibitors. Clin Lung Cancer. 2019;20:124-133e122.

\section{Publish your work in this journal}

Cancer Management and Research is an international, peer-reviewed open access journal focusing on cancer research and the optimal use of preventative and integrated treatment interventions to achieve improved outcomes, enhanced survival and quality of life for the cancer patient.
The manuscript management system is completely online and includes a very quick and fair peer-review system, which is all easy to use. Visit http://www.dovepress.com/testimonials.php to read real quotes from published authors. 